\title{
2D-Hydrodynamic Energy Model Including Avalanche Breakdown Phenomenon for Power Field Effect Transistors
}

\author{
M. ROUSSEAU* and J. C. DE JAEGER \\ Institut d'Electronique et de Microélectronique du Nord, Cité Scientifique - Avenue Poincaré - B.P. 69 - 59652 \\ Villeneuve d'Ascq Cédex - France
}

\begin{abstract}
A 2D-Hydrodynamic model is carried out to predict the breakdown voltage of microwave field effect transistors. The model is based on the conservation equations inferred from Boltzmann's transport equation, coupled with Poisson's equation. In order to take into account the channel avalanche breakdown, the charge conservation equations for electrons and holes are considered and a generation term is introduced. The set of equations is solved using finite difference and different computational methods have been tested to save computing time. The model allows us to obtain accurate predictions for power transistors considering a usual gate recess. Results are performed for pseudomorphic AlGaAs/InGaAs/GaAs HEMTs.
\end{abstract}

Keywords: Hydrodynamic modeling; Power field effect transistor; Breakdown voltage

\section{INTRODUCTION}

Breakdown phenomenon makes up one of the main limitations of Field Effect Transistors (FETs) for microwave power amplification. In order to improve the device breakdown voltage, different gate-recess topologies were developed. They are mainly based on a single gate recess or a double step gate recess which can be symmetrical or asymmetrical [1-3]. Consequently, the power transistor structure is very complicated. It depends on a lot of different technological parameters, such as the thickness of the cap layer and its doping concentration or the gate recess offset, and unknown parameters such as the surface potential (due to Fermi level pinning).

In this background, a physical model giving accurate predictions makes up a very useful tool in order to optimize the device and to curtail the fabrication cost. In order to investigate the performance of power FETs, the model must enable us to study the main limitations of the device, the breakdown voltage for example. In this paper, in order to study the phenomenon of avalanche breakdown, a two-dimensional hydrodynamic energy model has been carried out. This model, built up

\footnotetext{
*Corresponding author. Tel.: (33) 3201978 20, Fax: (33) 3201978 88, e-mail: michel.rousseau@iemn.univ-lille1.fr
} 
from previous versions achieved in our laboratory [4-5] has been strongly modified and improved to take the phenomenon of channel avalanche into account. The minority carriers are introduced and a generation term is considered. This leads to a more complex software including a larger set of equations to solve, and it needs appropriate numerical methods to save computing time. The behaviour of the device is analysed by means of the distribution of the different physical quantities. The model includes the physical limitations specific to submicron gate length devices and makes it possible to study the breakdown mechanisms and the breakdown voltage determination for gate recessed devices close to experimental manufacturing. Transistors based on different existing semiconductor components can be modeled, such as MESFETs, uniformly or $\delta$-doped HEMTs structures.

\section{MODEL DESCRIPTION}

The numerical model used in this study was described in detail in previous papers $[4,5]$. It is based on the conservation equations, derived from Boltzmann's equation, coupled with Poisson's equation. Our model is a Monte Carlo calibrated model, including a full two-dimensional treatment of the equations and the boundary conditions. Consequently, there is no simplifying assumption on the carrier energy. We are able to model structures very closed to experimental making: we take the real gate recess into account and we can consider complex devices (one or several $\delta$ doped layers, one or several channel in the structure).

In order to take the avalanche phenomenon into account, minority carriers are introduced in the model and a generation term is included in the charge conservation equations. The equations to be solved are the following ones:

- charge conservation equations for electrons and holes:

$$
\frac{\partial n}{\partial t}+\operatorname{div}\left(n \vec{v}_{n}\right)=G-R
$$

$$
\frac{\partial p}{\partial t}+\operatorname{div}\left(p \vec{v}_{p}\right)=G-R
$$

where $\mathrm{n}$ and $\mathrm{p}$ are the electron and hole carrier concentrations, $v_{n}$ and $v_{p}$ the carrier velocities. The recombination rate $\mathrm{R}$ is assumed to be zero, when the generation rate $G$ is written:

$$
G=\alpha_{n} n v_{n}+\alpha_{p} p v_{p}
$$

where $\alpha_{\mathrm{n}}$ and $\alpha_{\mathrm{p}}$ are the electron and hole impact ionization coefficients [6]:

$$
\alpha_{n, p}(F)=\frac{q F}{w_{i}} \exp \left[A-\sqrt{A^{2}+X^{2}}\right]
$$

$\mathrm{F}$ is the electric field, $\mathrm{A}$ and $\mathrm{X}$ are given by the following equations:

$$
A=0.217\left(\frac{w_{i}}{\left\langle w_{p}\right\rangle}\right)^{\gamma} \text { and } X=\frac{w_{i}}{q F \lambda}
$$

where $w_{i}$ is the ionization threshold energy, $q$ is the charge unit and $\lambda,\left\langle w_{p}\right\rangle$ are as follows:

$\lambda=\lambda_{0} \tanh \left(\frac{w_{p}}{2 k_{B} T_{e}}\right)$ and $\left\langle w_{p}\right\rangle=w_{p} \tanh \left(\frac{w_{p}}{2 k_{B} T_{e}}\right)$

In these expressions, $\mathrm{w}_{\mathrm{p}}$ is the optical phonon energy, $\lambda_{0}$ is the mean free path for optical phonon scattering at $0 \mathrm{~K}, \mathrm{k}_{\mathrm{B}}$ is Boltzmann's constant and $\mathrm{T}_{\mathrm{e}}$ is the electronic temperature.

- energy conservation equation for the electrons:

$$
\frac{\partial n w}{\partial t}=-q n \vec{v} \vec{F}-\nabla \vec{S}_{n}-\frac{n\left(w-(3 / 2) k_{B} T_{o}\right)}{\tau_{w}}-w G
$$

where $\mathrm{w}$ is the carrier energy, $\tau_{\mathrm{w}}$ is the energy relaxation time and $S_{n}$ is the energy flux:

$$
\vec{S}_{n}=-\kappa \vec{\nabla} T_{e}+\left[w+k_{B} T_{e}(w)\right] n \vec{v}
$$


where $\kappa$ is the thermal conductivity, given by the Wiedemann-Frantz-Law [7]:

$$
\kappa=\frac{5}{2}\left(\frac{k_{B}}{q}\right)^{2} q n \mu_{n} T_{e}
$$

The energy conservation equation for the holes is not considered. A drift diffusion behaviour is assumed for the minority carriers.

- carrier velocity equations:

$$
\begin{aligned}
& \vec{v}_{n}=\mu_{n}\left(-\vec{F}-\frac{1}{n} \vec{\nabla}\left(n k_{B} T_{e}\right)\right) \\
& \vec{v}_{p}=\mu_{p}\left(-\vec{F}-\frac{1}{p} \vec{\nabla}\left(n k_{B} T_{o}\right)\right)
\end{aligned}
$$

These equations are obtained by the momentum conservation equations where the inertial terms are neglected. This hypothesis is fully justified for gate length FETs larger than $0.5 \mu \mathrm{m}$ and enable us to solve a simplified set of equations.

- Poisson's equation

$$
\nabla(\varepsilon \vec{E})=q\left(N_{d}^{+}-n+p\right)
$$

where $N_{\mathrm{d}}^{+}$is the ionized donor concentration and $\varepsilon$ is the dielectric permittivity. The physical parameters such as $\mu_{\mathrm{n}}, \mu_{\mathrm{p}}, \tau_{\mathrm{w}}$ and $\tau_{\mathrm{m}}$ depend on the average total energy in the material. They are obtained from steady-state three valleys Monte Carlo simulations [8]. The main physical phenomena are taken into account and the whole carrier dynamics are treated, particularly the carrier transfer in the upper valleys, by means of a single equivalent electron gas [4].

The model can take into account a gate recess area close to experimental making. Appropriate boundary conditions are applied around the simulated area and inside the device at the semiconductor-insulator interface. Neumann boundary conditions are applied on the free surfaces so that no current flux leaves the simulated device area. Dirichlet boundary conditions are applied on the source and drain contacts $\left(\mathrm{T}_{\mathrm{e}}=\mathrm{T}_{\mathrm{o}}\right.$; $\mathrm{n}=N_{\mathrm{d}}^{+} ; \mathrm{V}=$ external bias). On the gate contact, the potential is raised by the value of the junction diffusion potential to take the Schottky barrier height into account. The insulator area is supposed to be ideal. Consequently, the orthogonal carrier velocity is equal to zero at the interface between the semiconductor and the insulator. The discontinuities of the conduction band are modeled using an equivalent transverse electric field at the interfaces and the surface potential effect is introduced using electrostatic charges in the gate recess area. The generation term depends on the carrier energy for the electrons and on the longitudinal electric field for the holes [8].

The equations are discretized using a finite difference method where the varibles used are the potential $\mathrm{V}$, the electron density $\mathrm{n}$, the hole density $\mathrm{p}$ and the electron energy w. A non uniform mesh is used in both directions. The scalar variables are defined at the mesh points whereas the vector quantities are defined at the half-points. Poisson's equation is discretized using a centered finite difference and is solved using the matrix double sweep method [10]. Carrier velocity equations for electrons and holes are discretized using an upwind method for stability reasons. In the charge conservation equations for electrons and holes and in the electron energy conservation equation, the spatial discretization is performed using a centered finite difference method and the temporal discretization is performed using a full implicit method. These three non-linear equations are linearized using a Newton-Raphson Method and solved from a Bi-CGSTAB algorithm [11]. A transient simulation is carried out up to the steady state determination. From this model, it is possible to obtain the distribution of the physical quantities and the current-voltage characteristics. The small signal parameters of the equivalent scheme are obtained either from incremental methods on $V_{G S}$ 
or $\mathrm{V}_{\mathrm{DS}}$ or from the response to a Heaviside step followed by FFT [12].

\section{RESULTS}

The model is used to study the performance of power AlGaAs/GaInAs/GaAs Pseudomorphic HEMTs manufactured by United Monolithic Semiconductor. The device is a symmetrical single gate recess structure with a GaInAs well (thickness $12.5 \mathrm{~nm})$, a top $\delta$-doped layer $\left(3 \times 10^{12} \mathrm{~cm}^{-2}\right)$ and a
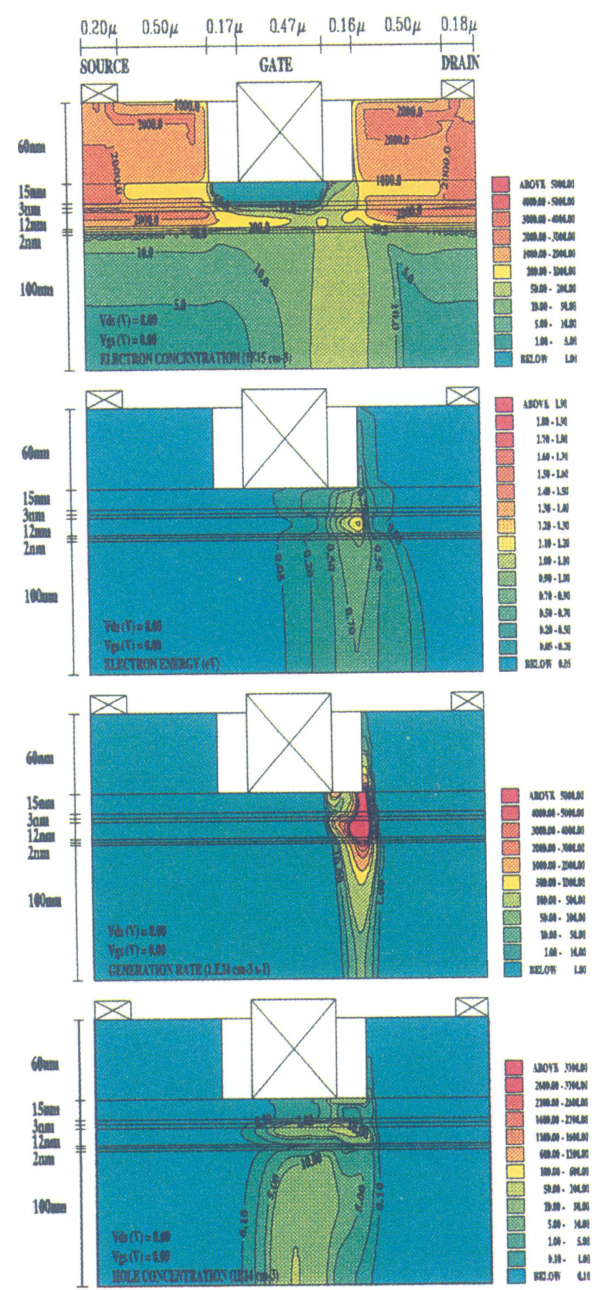

FIGURE 1 Physical quantities distribution for a pseudomorphic AlGaAs/InGaAs HEMT at open channel $\left(\mathrm{V}_{\mathrm{GS}}=0 \mathrm{~V}\right.$; $\mathrm{V}_{\mathrm{DS}}=8 \mathrm{~V}$ ). (See Color Plate V). bottom $\delta$-doped layer $\left(10^{12} \mathrm{~cm}^{-2}\right)$. The thickness of the various layers (Spacers, upside and backside barrier layers) and topology sizes are given in Figure 1.

The distribution of the physical quantities (electron and hole concentrations, electron energy and the avalanche generation rate) are represented in Figure 1 for bias conditions corresponding to device breakdown at open channel $\left(\mathrm{V}_{\mathrm{DS}}=8 \mathrm{~V}\right.$, $\left.\mathrm{V}_{\mathrm{GS}}=0 \mathrm{~V}\right)$. We can notice that the electron energy is maximum in the channel at the gate exit. Most of the holes drift towards the source and a small part towards the gate in the gate recess offset area
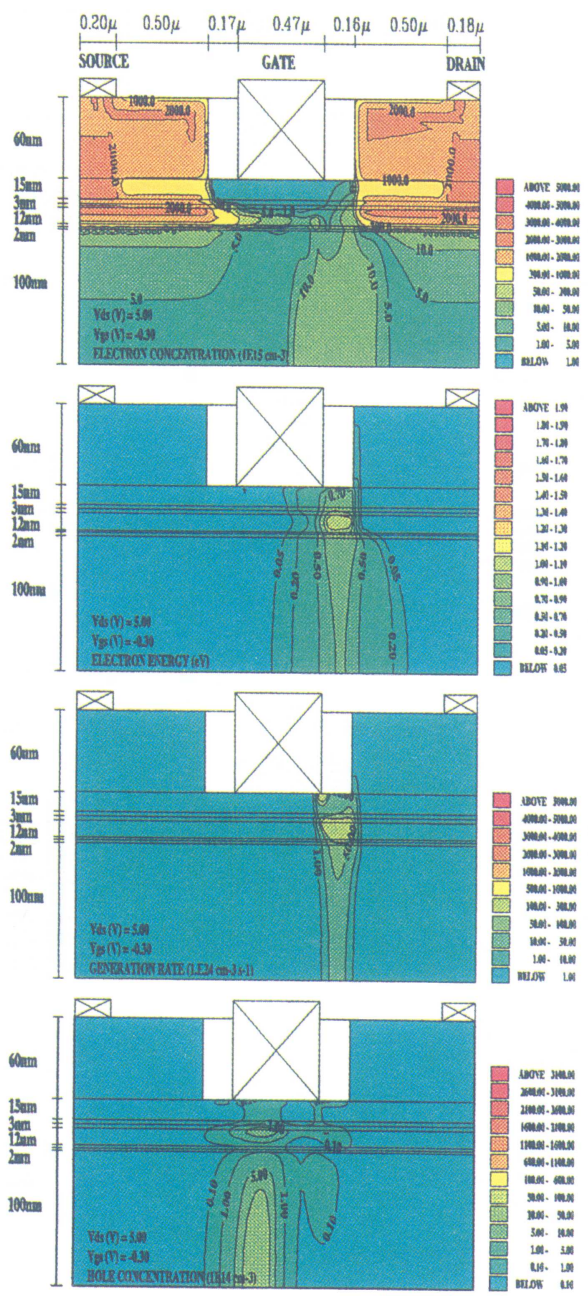

FIGURE 2 Physical quantities distribution for a pseudomorphic $\mathrm{AlGaAs} / \mathrm{InGaAs}$ HEMT close to pinchoff $\left(\mathrm{V}_{\mathrm{GS}}=\right.$ $\left.-0.3 \mathrm{~V} ; \mathrm{V}_{\mathrm{DS}}=8 \mathrm{~V}\right)$. (See Color Plate VI). 


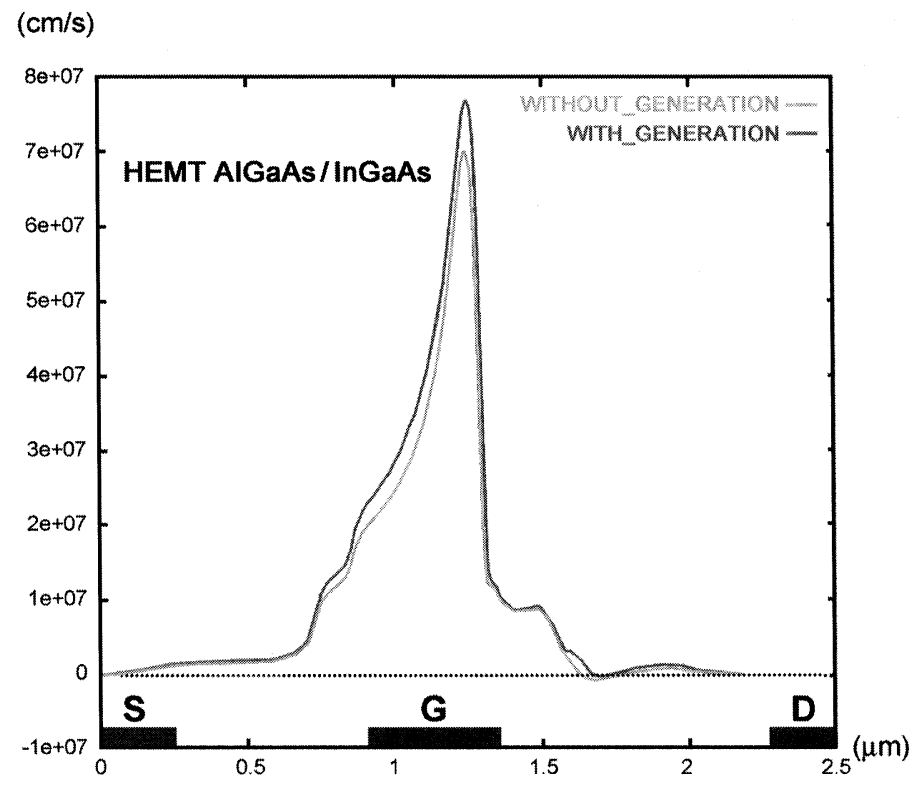

FIGURE 3 Average electron velocity along the structure.

where the electric field is high. The electron-hole carrier generation occurs at the place where the electron energy and the electric field are maximum at the exit of the gate. Figure 2 shows the same physical parameters close to pinchoff at the same drain-source bias $\left(\mathrm{V}_{\mathrm{DS}}=8 \mathrm{~V}, \mathrm{~V}_{\mathrm{GS}}=-0.3 \mathrm{~V}\right)$. In this case, the electron energy maximum is smaller as well as the avalanche carrier generation. This can be seen from the generation rate and the hole concentration distribution. This is due to a higher device breakdown voltage at pinchoff when compared to the one usually observed at open channel.

The average electron longitudinal velocity in the device is represented Figure 3 with and without (G assumed to be equal to zero) the generation phenomenon. An increase of the velocity is observed when the ionization phenomenon is introduced in the model. This is due to a drop in the carrier energy in the high energy area (Fig. 4) evolving from the energy lost by the carriers due to the avalanche multiplication.

Figure 5 shows the drain current $I_{D S}$, the transconductance $\mathrm{g}_{\mathrm{m}}$ and the gate-source capacitance $\mathrm{C}_{\mathrm{GS}}$ versus $\mathrm{V}_{\mathrm{GS}}$ for $\mathrm{V}_{\mathrm{DS}}=8 \mathrm{~V}$. When the ionization phenomenon is taken into account, a
(eV)

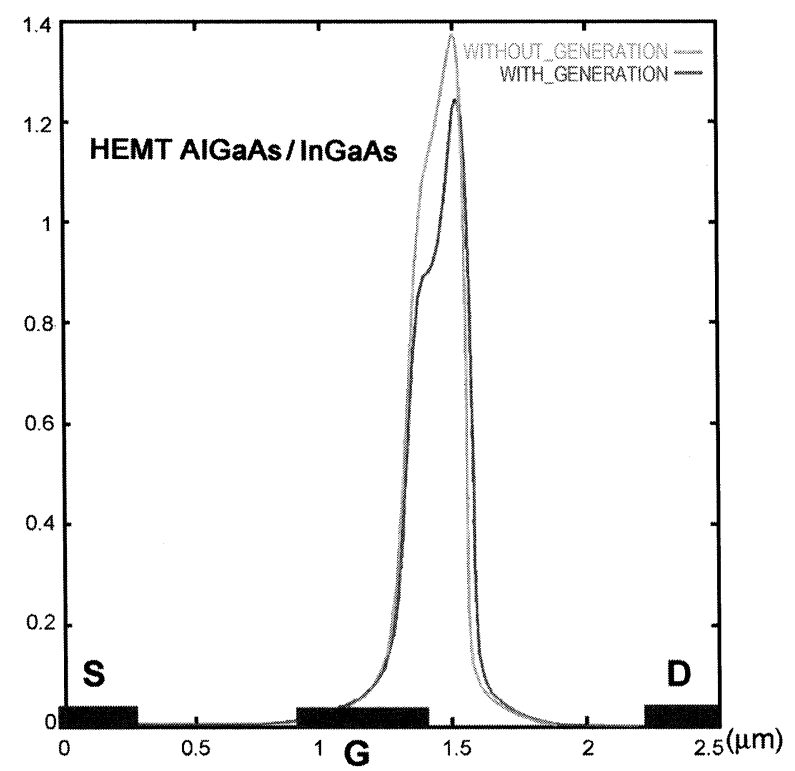

FIGURE 4 Average electron energy along the structure.

higher transconductance is obtained due to the current increase. The $\mathrm{C}_{\mathrm{GS}}$ capacitance remains practically identical. 

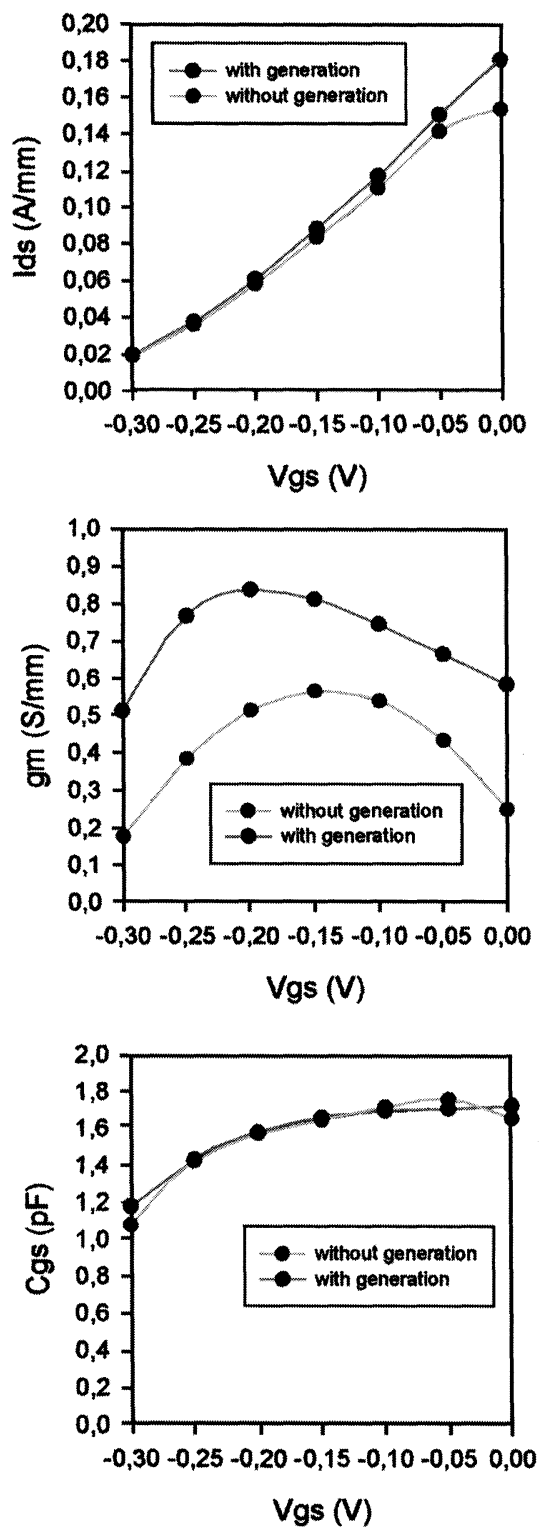

FIGURE 5 Drain current, transconductance and gate-source capacitance versus $\mathrm{V}_{\mathrm{GS}}\left(\mathrm{V}_{\mathrm{DS}}=8 \mathrm{~V}\right)$.

\section{CONCLUSION}

The reported 2D hydrodynamic model helps understand the avalanche breakdown physical behaviour of microwave power field effect transistors by means of the study of the device physical quantities. And it especially shows the electron and hole distributions as well as the generation rate and its influence on the carrier velocity and carrier energy. The breakdown voltage of the device drawn from the current-voltage characteristic strongly depends on these parameters. So, the model makes up a useful tool for the optimization of the breakdown voltage of power devices considering various gate recess topologies such as for instance a double step gate recess. In this background, due to the device complexity and the large number of technological parameters to be taken into account, it makes up a useful tool to help the designers and to curtail the development costs.

\section{References}

[1] Lee, J. L., Kim, H., Mun, J. K. and Maeng, S. J., "A Kuband T-shaped gate GaAs power MESFET with high breakdown voltage for satellite communications", IEEE Electron Device Letters, July, 1998.

[2] Gaquiere, C., Bonte, B., Theron, D., Crosnier, Y., ArseneHenri, P. and Pacon, T., "Breakdown analysis of an asymmetrical double resseced power MESFET's", IEEE Trans. Electron Devices, February, 1995.

[3] Lu, S. S., Meng, C. C., Lin, Y. S. and Lan, H., "The effect of gate recess profile on device performance of $\mathrm{Ga}_{0.51}$ $\mathrm{In}_{0.49} \mathrm{P} / \mathrm{In}_{0.2} \mathrm{Ga}_{0.8}$ As doped-channel FET's", IEEE Trans. Electron Devices, January, 1999.

[4] Shawki, T., Salmer, G. and El-Sayed, O., "MODFET 2-D hydrodynamic energy modeling: optimization of subquarter-micron-gate structures", IEEE Trans. Electron Devices, January, 1990.

[5] Delemer, J. D., Rousseau, M., De Jaeger, J. C. and Lefebvre, M. (1997). "Recent improvements of 2-D hydrodynamic model for HEMT simulations", Proc. of $10^{\text {th }}$ III-V Semiconductor Device Simulation Workshop.

[6] Chau, H. F. and Pavlidis, D., "A physics-based fitting and extrapolation method for measured impact ionization coefficients in III-V semiconductors", J. Appl. Phys., July, 1992.

[7] Simlinger, T., Brecht, H., Grave, T. and Selberherr, S., "Simulation of Submicron Double-Heterojunction High Electron Mobility Transistors with MINIMOS-NT", IEEE Trans. Electron Devices, May, 1997.

[8] Jacobini, C. and Reggiani, L., "The Monte Carlo method for the solution of charge transport in semiconductors with applications to covalent materials", Rev. Mod. Phys., July, 1983.

[9] Souissi, K., Odeh, F., Tang, H., Gnudi, A. and Lu, P. F., "Investigation of the impact ionization in the hydrodynamic model", IEEE Trans. Electron Devices, August, 1993.

[10] Ibrahim, M., "Two-dimensional simulation of microwave GaAs submicronic-gate FETs", Thesis, Cairo University, 1983.

[11] Van der Vorst, H. A., "Bi-CGSTAB: A fast and smoothly non symetric linear systems", J. Sci. Stat. Comput., March, 1992.

[12] Laux, S. E., "Techniques for small-signal analysis of semiconductor devices", IEEE Trans. Electron devices, 1985. 

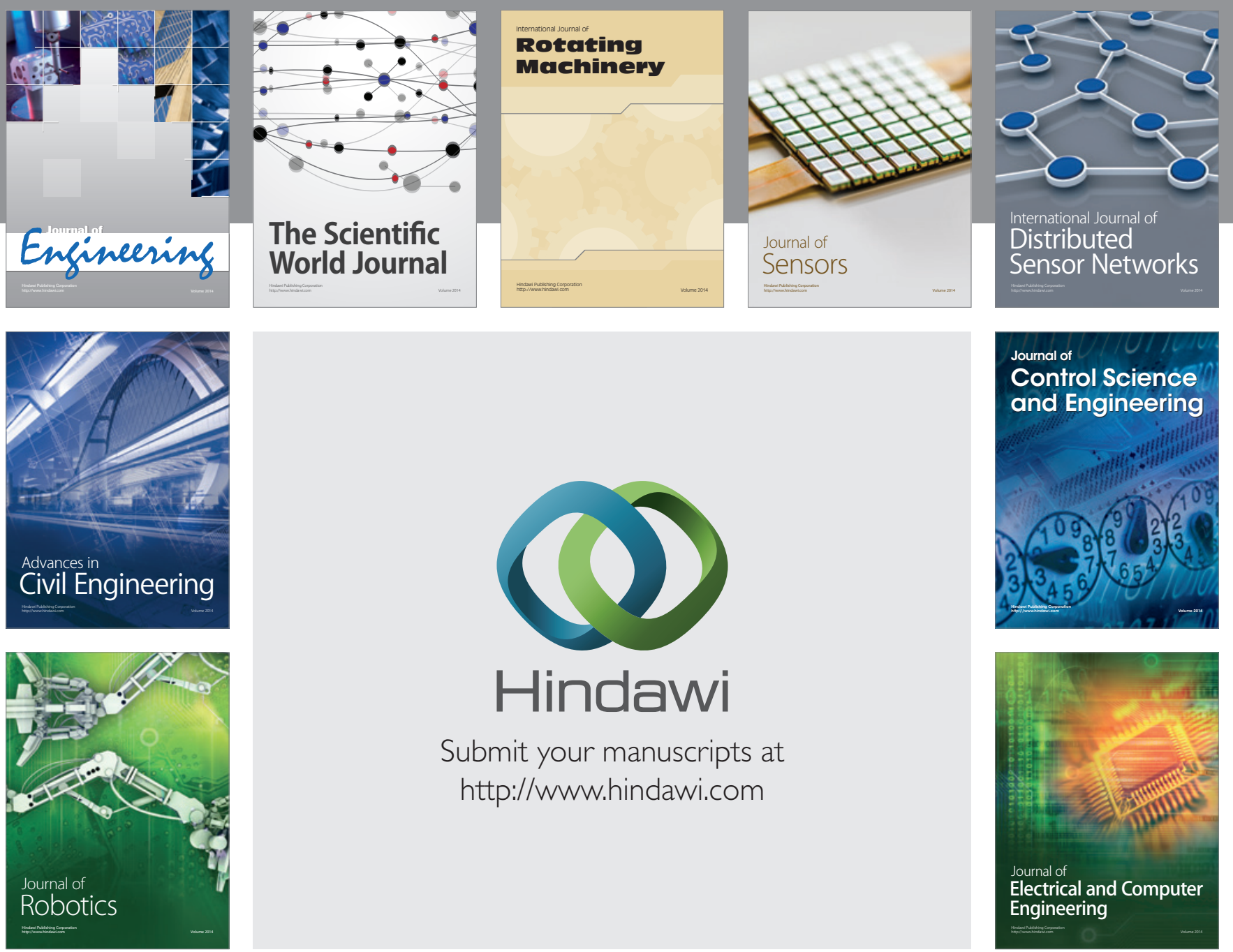

Submit your manuscripts at

http://www.hindawi.com
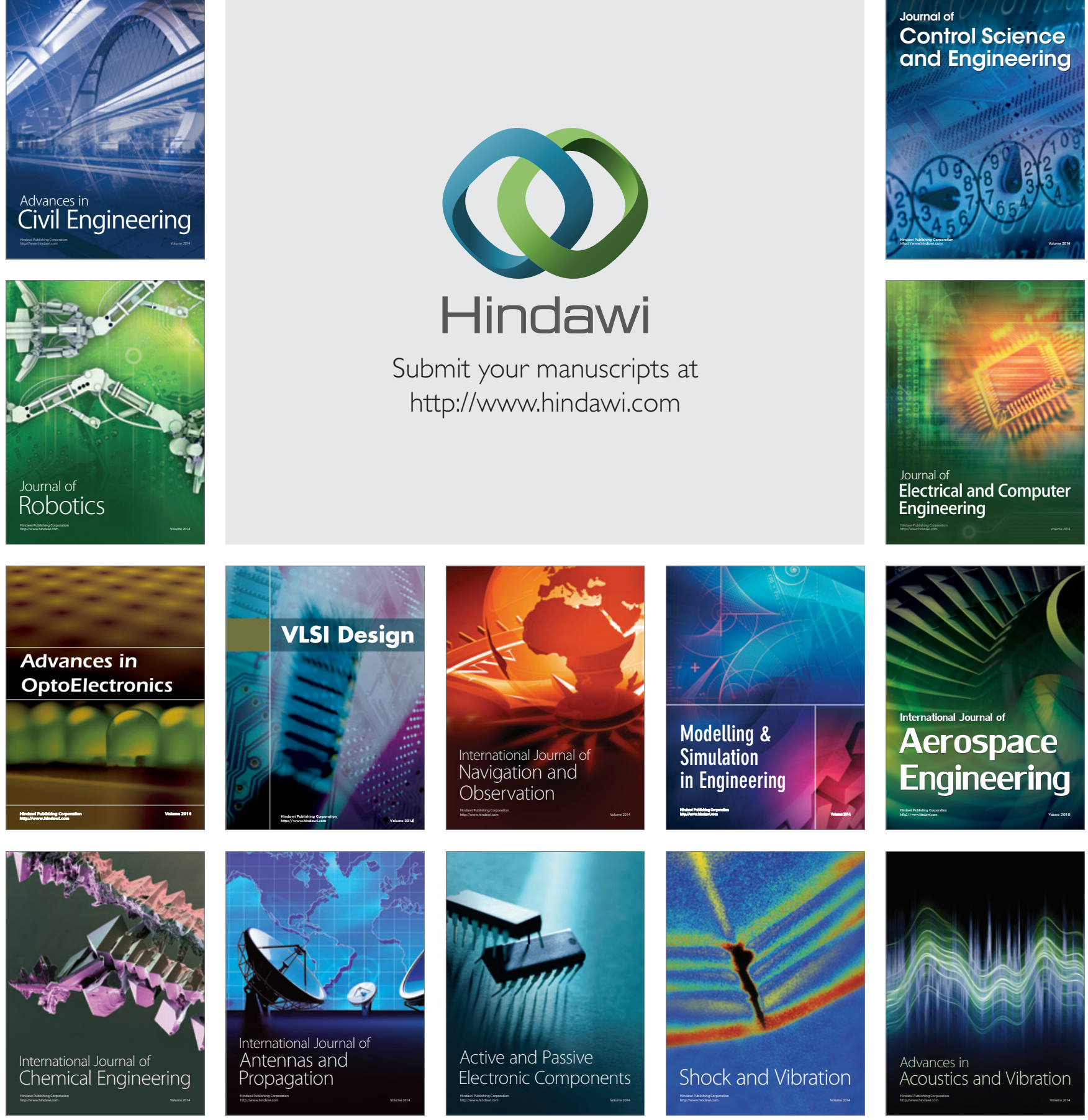